\title{
Michelson \\ DIAGNOSTICS
}

\section{In vivo real-time optical coherence tomography imaging of Drosophila for cardiovascular research}

\begin{abstract}
Optical coherence tomography (OCT) is a powerful imaging modality. OCT scanners, such as the Michelson Diagnostics EX1301 OCT microscope, uniquely provide real-time, in vivo, micrometer-scale images of biological tissue to a depth of $1 \mathrm{~mm}$ or more, allowing noninvasive study of dynamic tissue microstructure of living organisms at depths beyond the reach of standard microscopy. Here we describe the use of OCT to study the cardiovascular dynamics of Drosophila melanogaster.
\end{abstract}

Optical coherence tomography (OCT)-pioneered at the Massachusetts Institute of Technology in 1991 (ref. 1)—has been successfully commercially applied to imaging the retina and is now finding applications in other fields. Briefly, OCT imaging involves scanning tissue with a focused laser beam, detecting the backscattered light at the same wavelength and using an interferometer to select only the light that has not been multiply scattered. This light can be used to reconstruct an image with good contrast at depths of $1 \mathrm{~mm}$ or more in tissue that is opaque to conventional light microscopy. In 1997, Fourier-domain OCT (FD-OCT) was first proposed ${ }^{2}$; it enabled much faster scanning and improved signal-to-noise ratio, producing real-time, detailed images in more highly scattering tissue than the retina.

A limitation with FD-OCT is that the lateral resolution achievable is limited by the numerical aperture of the laser beam: at 1,300 nm a depth of focus of $1 \mathrm{~mm}$ limits the lateral resolution to $15-20 \mu \mathrm{m}$. To overcome this, Michelson Diagnostics Ltd developed 'Multi-beam $\mathrm{OCT}^{\prime}$, which uses four beams focused at slightly different depths to capture a 'mosaic' OCT image with twice the resolution of a singlebeam OCT system ${ }^{3}$. The equipment used in the application presented here is a Michelson Diagnostics Ltd EX1301 OCT Microscope (Fig. 1). This multi-beam OCT scanner was fitted with an objective lens that produced a $0.5 \mathrm{~mm}$ depth of focus and $<5 \mu \mathrm{m}$ lateral resolution. A motorized stage was used to translate the subject under the scan so as to build up three-dimensional images.

\section{OCT imaging of Drosophila}

The Drosophila 'heart', or dorsal vessel, is a linear tube that helps propel hemolymph through the fly's open circulatory system. Since the discovery that the transcription factors Tinman and its vertebrate

\section{Jon Holmes}

Michelson Diagnostics Ltd, Orpington, Kent, UK. Correspondence should be addressed to J.H. (jon.holmes@md-Itd.co.uk).

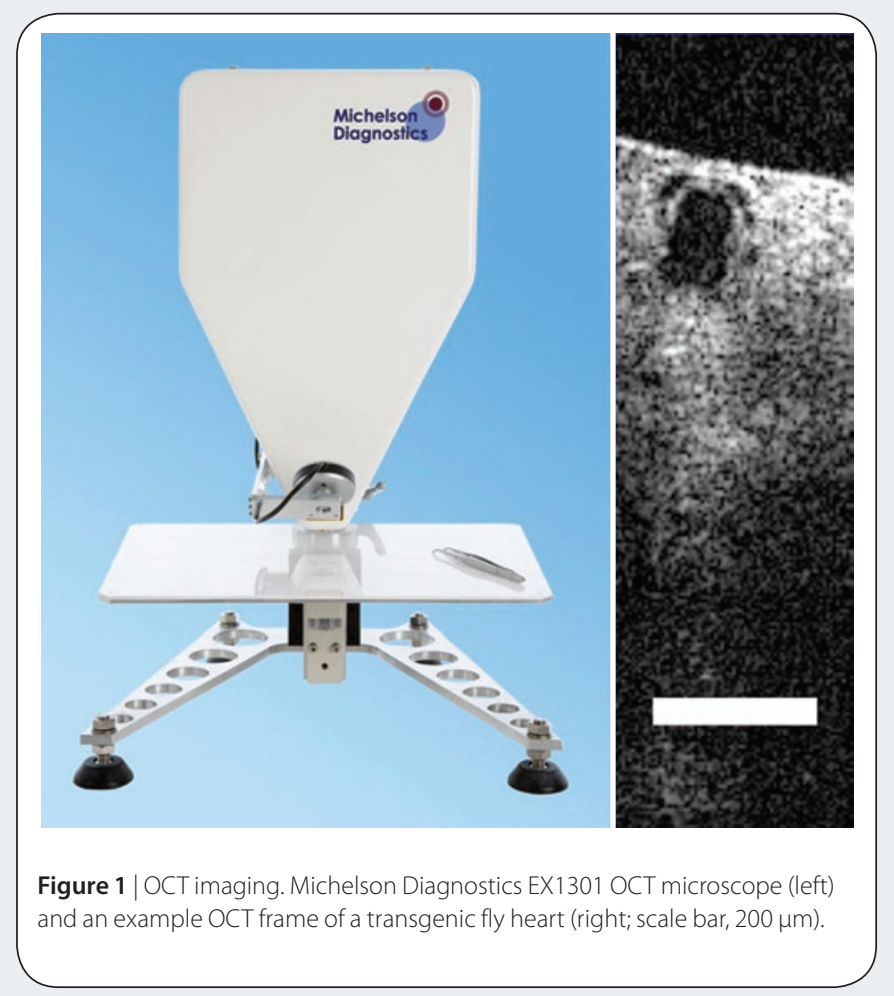

NKX homologs direct heart development through evolutionarily conserved pathways ${ }^{4,5}$, Drosophila has been an important model for the study of cardiac development. The functional and molecular parallels between fly and human hearts have prompted several laboratories to take advantage of the available tools for complex genetic manipulation in Drosophila to advance genetic studies relating to human heart disease, including disturbances of heart rate, rhythm, remodeling and contractile function $^{6-9}$. OCT provides a rapid and noninvasive method to perform these studies of heart tube function in live and intact flies.

We used a gel plate with an immobilized live fly specimen under the OCT microscope, positioned so that the scan was across the 

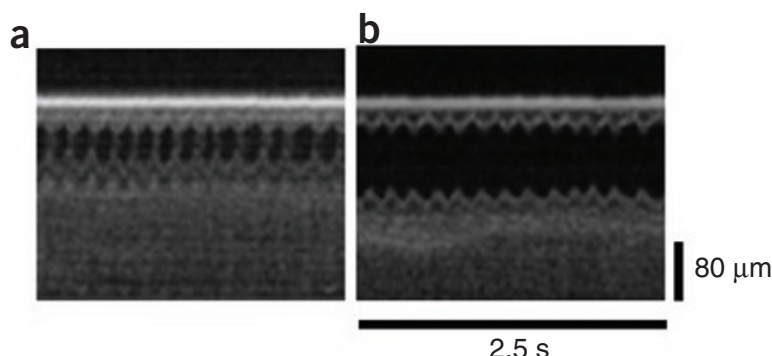

C

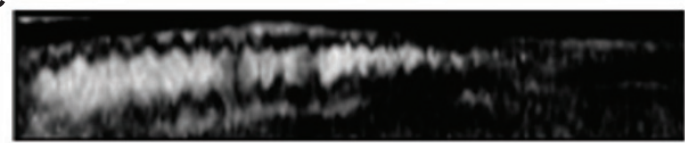

d

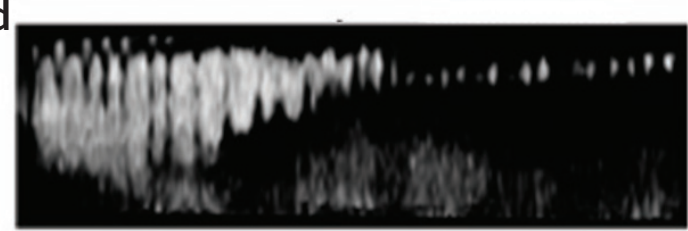

Figure 2 | Cardiomyopathic defects imaged by OCT. (a,b) M-mode images of the heart tube in a wild-type fly (a) and a transgenic fly in which a gene important for heart tube function has been knocked down by RNAi (b), producing a cardiomyopathic phenotype with increased heart tube dilation and decreased cardiac contractility. (c,d) Side-view snapshot of a four-dimensional image from wild-type flies (c) and flies with a cardiomyopathy induced by RNAi (d). Anterior is to the left.

heart tube. Typically, in each scan, we collected 400 frames of 200 $\mu \mathrm{m}$ width at a frame rate of 39.3 frames per second, which is sufficient to resolve the heartbeat of $\sim 5$ beats per second. Using ImageJ software (US National Institutes of Health), M-mode images were generated from these frame stacks. Three representative heart tube contractions were selected from a region that showed the predominant heart phenotype, and the end systolic diameter (ESD) and end diastolic diameter (EDD) were measured and used to calculate the average ESD, EDD and percentage fractional shortening (a measure of heart tube contractility).

To visualize the fly heart, we created four-dimensional images by scanning with the specimen moving in the direction of the heart tube at a velocity of $0.05 \mathrm{~mm} \mathrm{~s}^{-1}$, thus capturing a sequence of frames with a spatial separation close to $1.25 \mu \mathrm{m}$ and a temporal separation of $25 \mathrm{~ms}$. The TIF image stack was then cropped, processed with the despeckle and smooth function, intensity-inverted to make the heart tube bright and surrounding tissue dark, and displayed using the Voxx (Indiana Center for Biological Microscopy) rendering program.

We used the OCT microscope to scan wild-type flies as well as flies expressing an RNA interference (RNAi) construct to knock down expression of a gene important for heart tube function. The microscope easily detected the cardiomyopathic phenotype, as the M-mode images showed an increased heart tube diameter (Fig. 2a,b) and decreased contractility in flies with the RNAi-induced cardiomyopathy. A fourdimensional image showed the increase in dilation in cardiomyopathic

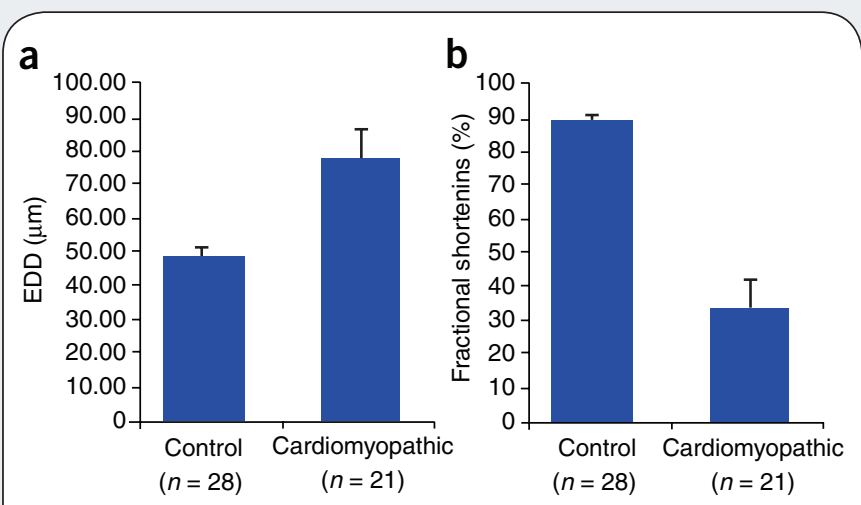

Figure 3 ESD and percentage fractional shortening in cardiomyopathic flies. (a,b) Graphs showing the increase in cardiac ESD $(\mathbf{a})$ and the decrease in fractional shortening $(\mathbf{b})$ in cardiomyopathic flies when compared to wild-type controls.

hearts and indicated whether this was confined to particular regions of the heart tube (Fig. 2c,d). The dilation of the heart tube in these cardiomyopathic flies was most obvious at the anterior of the heart tube (Fig. 2c,d). The ESD and percentage fractional shortening were also quantifiably different between wild-type and cardiomyopathic hearts (Fig. 3).

\section{Conclusion}

The Michelson Diagnostics OCT microscope is a valuable tool that allows easy measurement of important cardiac parameters in adult Drosophila flies of different genotypes. The commercial availability of this instrument will allow the power of Drosophila genetics and the simplicity of this biological system to be harnessed to ask important questions about cardiac biology.

Additional information is available at http://www.michelson diagnostics.com/.

\section{ACKNOWLEDGMENTS}

I thank M. Snee from G. Dorn's laboratory at the Washington University School of Medicine, St. Louis, for his assistance.

1. Huang, D. et al. Optical coherence tomography. Science 254, 1178-1181 (1991).

2. Fercher, A.F. et al. Measurement of intraocular distances by backscattering spectral interferometry. Opt. Commun. 117, 43-48 (1995).

3. Holmes, J. et al. Multichannel Fourier domain OCT system with superior lateral resolution for biomedical applications. Proc. SPIE 6847,684700 (2008).

4. Bodmer, R. Heart development in Drosophila and its relationship to vertebrate systems. Trends Cardiovasc. Med. 5, 21-27 (1995).

5. Harvey, R.P. NK-2 homeobox genes and heart development. Dev. Biol. 2, 203216 (1996).

6. Allikian, M. et al. Reduced life span with heart and muscle dysfunction in Drosophila sarcoglycan mutants. Hum. Mol. Genet. 16, 2933-2943 (2007).

7. Taghli-Lamallem, O. et al. Dystrophin deficiency in Drosophila reduces lifespan and causes a dilated cardiomyopathy phenotype. Aging Cell 7, 237-249 (2008).

8. Wolf, M. et al. Drosophila as a model for the identification of genes causing adult human heart disease. Proc. Natl. Acad. Sci. U.S.A. 5, 1394-1399 (2006).

9. Ocorr, K. et al. KCNQ potassium channel mutations cause cardiac arrhythmias in Drosophila that mimic the effects of aging. Proc. Natl. Acad. Sci. U.S.A. 104, 3943-3948 (2007).

This article was submitted to Nature Methods by a commercial organization and has not been peer reviewed. Nature Methods takes no responsibility for the accuracy or otherwise of the information provided. 